\title{
Model calculations of the ionization relaxation and radiative cooling in unsteady krypton and xenon shock waves
}

\author{
By D. KRAUSS-VARBAN AND F. DEMMIG \\ Institut für Plasmaphysik, Universität Hannover, \\ Callinstraße 38, 3000 Hannover 1, West Germany
}

(Received 15 February 1984)

The ionization relaxation and radiative cooling of weakly unsteady shock waves in krypton and xenon is examined. We find that, besides radiation energy loss, the shock-tube boundary layers and shock attenuation strongly influence the radiativecooling region. Calculations taking these effects into account are in excellent agreement with experimental values of electron densities. The contribution of line radiation to total radiation energy loss is obtained, and amounts to about $54 \%$ at temperatures of $10000 \mathrm{~K}$ in krypton. For xenon, first comparisons indicate a similar result.

\section{Introduction}

There have been numerous investigations of the radiative cooling behaviour of shock waves in inert gases (e.g. Oettinger \& Bershader 1967; Horn, Wong \& Bershader 1967; Pinègre \& Valentin 1974; Glass \& Liu 1978; Vaguin et al. 1978 in argon; Rehder et al. 1969; Glass, Liu \& Tang 1977 in krypton; Enomoto 1972 in xenon). Considerable effort has been made in calculating the line-radiation energy loss and in employing reabsorption models (Horn et al. 1967; Enomoto 1972). Frequently, calculations do not include the relaxation zone, but are started at the beginning of the radiative-cooling region. Despite this simplification, only rough agreement has been obtained, in many cases, between theoretical and experimental results of electron-density values. This can be caused by neglecting or simplifying the effects of boundary layers and shock attenuation.

The aim of this paper is to treat continuum and line radiation in a concise manner (see $\$ 2$ ), and to maintain, as far as possible, high accuracy in the case of weakly unsteady shock waves of medium Mach numbers (8-12) in krypton and xenon.

To achieve this, use is made of the gasdynamical equations for unsteady flow, including a boundary-layer term as well as the radiative energy loss. The time history of the particle densities in the shock-tube plasma is described by a two-level model. Details of the calculation are given in $\$ 3$. In $\$ 4$ computed electron densities are compared with experimental values for krypton and xenon shock waves.

\section{Computation of radiation energy loss}

It is well known that, besides collisional processes, radiative effects play an important role in non-equilibrium ionization. For medium Mach numbers and in the range of density considered here (upstream density $0.02-0.1 \mathrm{~kg} / \mathrm{m}^{3}$ ) effects of the 
optically thick, photoionizing continuum radiation can be neglected (Krauss-Varban 1981). Also, resonant line radiation - in comparison with collisional processes - gives rise to appreciable rates of excitation only ahead of the shock front. Far more important is the energy loss due to optically thin continuum and line radiation. In addition to boundary-layer effects and shock attenuation, radiation cooling determines the downstream behaviour of the electron density investigated here.

The contributions to the radiation energy loss per unit volume and time $(Q)$ are due to radiative recombination $\left(Q_{\mathrm{fb}}\right)$ and bremsstrahlung in the field of the ions $\left(Q_{\mathrm{ff}}^{(\mathrm{i})}\right)$, free-free transitions in the field of the atoms $\left(Q_{\mathrm{ff}}^{(\mathrm{a})}\right)$ and line radiation $\left(Q_{\mathrm{L}}\right)$ :

$$
Q=Q_{\mathrm{fb}}+Q_{\mathrm{ff}}^{(\mathrm{i})}+Q_{\mathrm{ff}}^{(\mathrm{a})}+Q_{\mathrm{L}}
$$

In the Kramers--Unsöld theory the energy loss due to continuum radiation in the field of the ions (see e.g. Horn et al. 1967) is described by

$$
Q_{\mathrm{fb}}+Q_{\mathrm{ff}}^{(\mathrm{i})}=A \frac{n_{\mathrm{e}}^{2}}{T_{\mathrm{e}}^{\frac{1}{2}}}\left[Z_{\mathrm{eff}}^{2}\left(\nu_{\mathrm{c}}+\frac{k T_{\mathrm{e}}}{h}\right)\right]
$$

where $A=6.842 \times 10^{-51} \mathrm{~J} \mathrm{~m}^{3} \mathrm{~K}^{\frac{1}{2}} \cdot n_{\mathrm{e}}$ and $T_{\mathrm{e}}$ are the electron density and temperature, $k$ and $h$ are Boltzmann's and Planck's constants respectively, $Z_{\text {eff }}$ is an effective nuclear charge and $\nu_{\mathrm{c}}$ is the cut-off frequency.

A more exact and physically justified description is obtained by quantummechanical calculations, the results of which are presented in terms of the temperature- and frequency-dependent $\xi$-factor (see e.g. Hofsäß 1978). The emission coefficient $\epsilon_{v}$ reads

where

$$
\epsilon_{\nu} \mathrm{d} \nu=\frac{A}{4 \pi} \frac{n_{\mathrm{e}}^{2}}{T_{\mathrm{e}}^{\frac{1}{2}}} \xi\left(\nu, T_{\mathrm{e}}\right) \mathrm{d} \nu
$$

$$
\xi\left(\nu, T_{\mathrm{e}}\right)=\frac{g^{+}}{u_{+}(T)} \xi^{\mathrm{rb}}\left(\nu, T_{\mathrm{e}}\right)\left[1-\exp \left(\frac{-h \nu}{k T_{\mathrm{e}}}\right)\right]+\xi^{\mathrm{ff}}\left(\nu, T_{\mathrm{e}}\right) \exp \left(\frac{-h \nu}{k T_{\mathrm{e}}}\right)
$$

$g^{+}$is the statistical weight of the ions in the ground state, and $u_{+}$is the partition function of the ions.

To obtain an expression equivalent to (2), we integrated (4) numerically, utilizing the $\xi$-factors $\xi^{\mathrm{fP}}$ and $\xi^{\mathrm{fb}}$ (excluding the ionizing part) calculated by Hofsäß (1978 and personal communication). As a very good approximation, the integrated $\xi$-factor can be deseribed by a linear temperature dependence, similar to the expression shown in brackets in (2):

where

$$
Q_{\mathrm{fb}}+Q_{\mathrm{ff}}^{(\mathbf{i})}=A \frac{n_{\mathrm{e}}^{2}}{T_{\mathrm{e}}^{\frac{1}{2}}} \phi_{1}\left(\phi_{2}+\frac{k_{t_{\mathrm{e}}}^{m}}{h}\right)
$$

$$
\phi_{1}=2.11, \quad h \phi_{2}=1.95 \mathrm{eV} \quad \text { for krypton }
$$

and

$$
\phi_{1}=2.70, \quad h \phi_{2}=2.14 \mathrm{eV} \text { for xenon. }
$$

Using the symbol $\tilde{\kappa}(\nu)$ for the absorption coefficient (without induced emission) per density of electrons $\left(n_{\mathrm{e}}\right)$ and atoms $\left(n_{\mathrm{a}}\right)$, the radiation energy loss due to free-free transitions in the field of the atoms can be written as

$$
Q_{\mathrm{ff}}^{(\mathrm{a})}=\int 4 \pi \epsilon_{\nu} \mathrm{d} \nu=4 \pi n_{\mathrm{e}} n_{\mathrm{a}} \frac{2 h}{c^{2}} \int \exp \left(-\frac{h \nu}{k T_{\mathrm{e}}}\right) \nu^{3} \tilde{\kappa}(\nu) \mathrm{d} \nu
$$




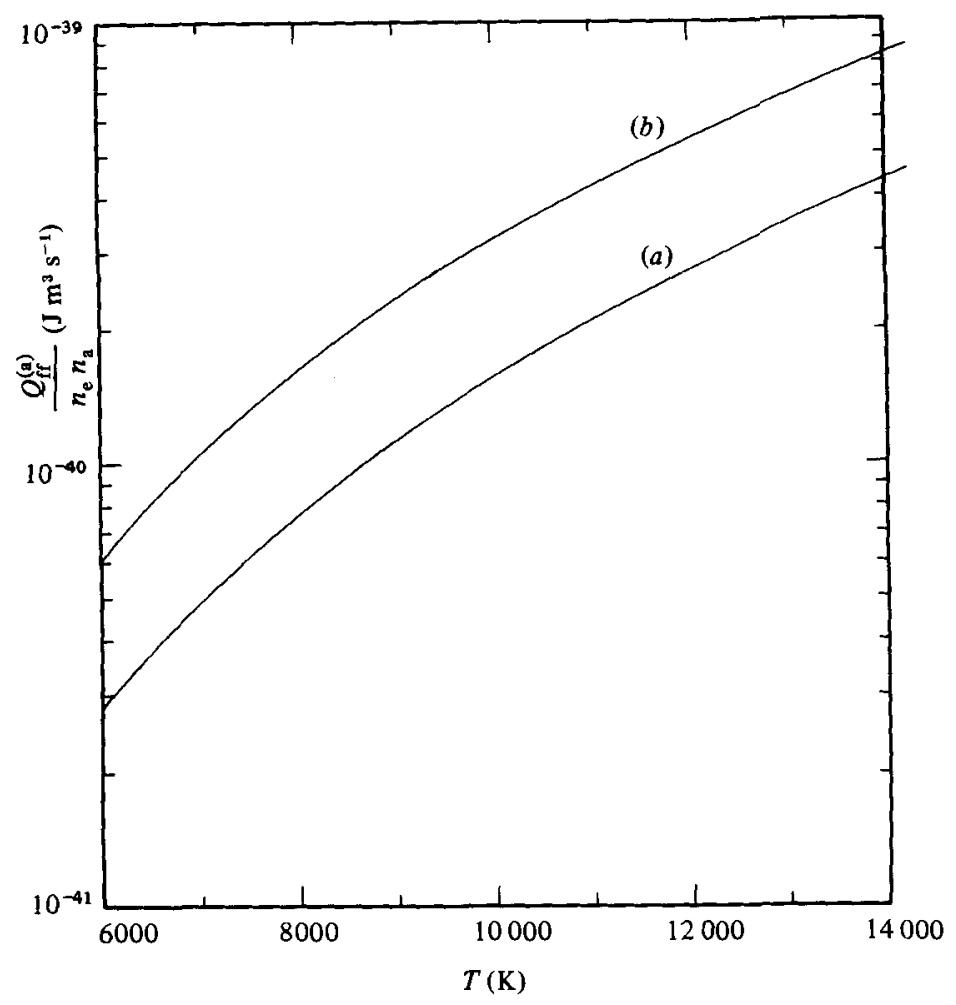

Figure 1. Radiation energy loss due to free-free transitions in the field of the atoms (normalized to unit density of electrons and atoms). The curves $(a)$ and $(b)$ correspond to krypton and xenon respectively.

We numerically integrated (6) with theoretical values of $\tilde{\kappa}\left(\nu, T_{\mathrm{e}}\right)$ calculated by Geltman (1973). No values were given for wavelengths $\lambda<0.5 \mu \mathrm{m}$, resulting in an estimated uncertainty for $Q_{\mathrm{ff}}^{(\mathrm{a})}$ of about $30 \%$ at $9500 \mathrm{~K}$, which equals the theoretical error of the values for $\lambda \geqslant 0.5 \mu \mathrm{m}$. However, these uncertainties are not crucial, if the contribution of $Q_{\mathrm{ff}}^{(\mathrm{a})}$ to total continuum energy loss does not exceed $20 \%$ in the radiative-cooling region, which is the case in the temperature and density ranges considered here. The results are shown in figure 1.

Horn et al. (1967) and Enomoto (1972) obtained an expression for the energy loss of line radiation $Q_{\mathrm{L}}$ by summing over all contributing transitions and assuming a model for reabsorption. Because of the uncertainties in transition probabilities, it is questionable whether the considerable additional effort is justified. Instead, we follow Glass et al. (1977), and make use of an adjustable factor for the contribution of line radiation:

where

$$
Q=Q_{\text {cont }}(1+a)
$$

$$
Q_{\mathrm{cont}}=Q_{\mathrm{fb}}+Q_{\mathrm{ff}}^{(\mathrm{i})}+Q_{\mathrm{ff}}^{(\mathrm{a})} .
$$

In contrast with absolute values, one can obtain the relative temperature dependence $a(T)$ with little effort: in local thermodynamic equilibrium (LTE) this temperature dependence results mainly from the proportionality to the respective upper-level density, as opposed to the proportionality to $n_{\mathrm{e}}$ in the case of the continuum. By making simple assumptions about the contributions of the different multiplets (here the results found for argon (Horn et al. 1967) have been transferred to krypton), one 


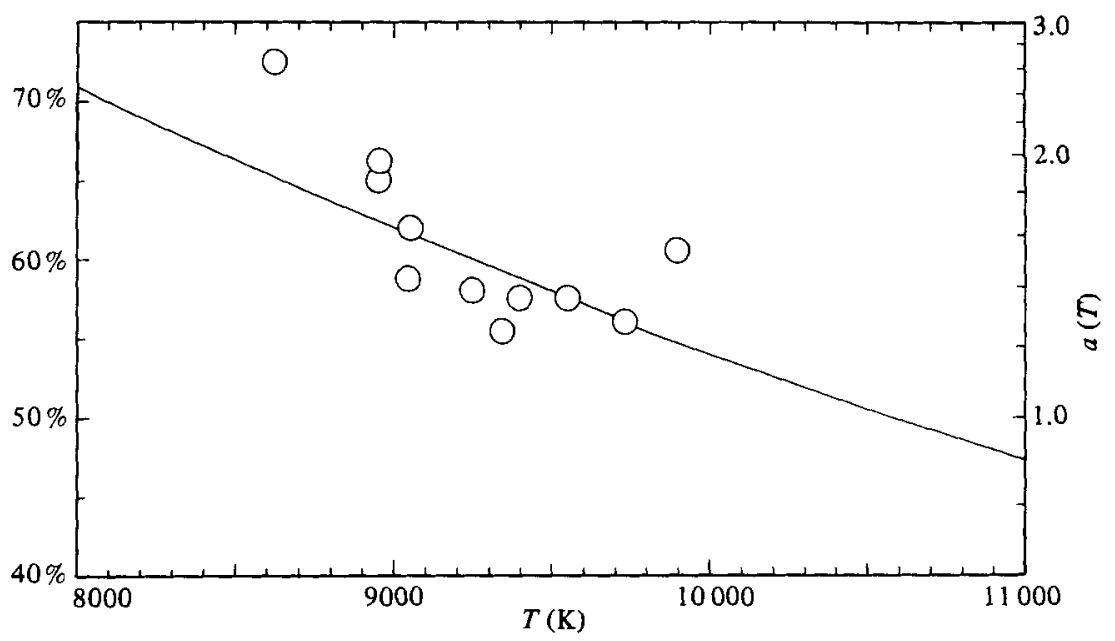

FigurE 2. Temperature dependence $a(T)$ of the contribution of line radiation. Left seale: percentage of total radiation energy loss. The circles denote some values of $a$ obtained by fitting computed to experimental electron densities in the radiative-cooling region (see \$4).

obtains a temperature dependence shown in figure 2. The curve is displayed neglecting a small correction term due to the different $n_{\mathrm{e}}$ dependence of $Q_{\mathrm{ff}}^{(a)}$; additionally, some experimental values have been indicated (see \$4).

For the limited temperature range occurring in the radiative-cooling region, a constant value of $a$ can be used to a reasonable degree of accuracy.

\section{Governing equations and numerical methods of solution}

Since we are mainly interested in electron densities, we make use of the well-known two-level model in describing the krypton and xenon energy levels. Uniting the four lowest excited levels and averaged with the statistical weights, the excited model-level is given mean energies of $10.18 \mathrm{eV}$ (krypton) and $8.359 \mathrm{eV}$ (xenon).

As usual, because of the high threshold energy, rates for direct ionization from the ground state are neglected. Consequently the rate equations read

$$
\left.\begin{array}{c}
\rho \frac{\mathrm{D}_{0}}{\mathrm{D} t}\left(\frac{n_{1}}{\rho}\right)=\dot{n}_{1(\mathrm{e}, \mathrm{a}, \mathrm{r})}=k_{01}^{\mathrm{a}} n_{0}^{2}-\left(k_{10}^{\mathrm{a}}+k_{1+}^{\mathrm{a}}\right) n_{0} n_{1}+k_{+1}^{\mathrm{a}} n_{0} n_{\mathrm{e}}^{2} \\
\quad+k_{01}^{\mathrm{e}} n_{0} n_{\mathrm{e}}-\left(k_{10}^{\mathrm{e}}+k_{1+}^{\mathrm{e}}\right) n_{\mathrm{e}} n_{1}+k_{+1}^{\mathrm{e}} n_{\mathrm{e}}^{3}+k_{+1}^{\mathrm{r}} n_{\mathrm{e}}^{2} \\
\rho \frac{\mathrm{D}_{0}}{\mathrm{~d} t}\left(\frac{n_{\mathrm{e}}}{\rho}\right)=\dot{n}_{\mathrm{e}(\mathrm{e}, \mathrm{a}, \mathrm{r})}=k_{1+}^{\mathrm{a}} n_{0} n_{1}-k_{+1}^{\mathrm{a}} n_{0} n_{\mathrm{e}}^{2}+k_{1+}^{\mathrm{e}} n_{\mathrm{e}} n_{1}-k_{+1}^{\mathrm{e}} n_{\mathrm{e}}^{3}-k_{+1}^{\mathrm{r}} n_{\mathrm{e}}^{2}
\end{array}\right\}
$$

In the rate coefficients $k$ of excitation and ionization, the indices $\mathrm{e}, \mathrm{a}$ and $\mathrm{r}$ denote collisions with electrons and atoms respectively and radiation. The subscripts $0,1,+$ and e refer to the ground state, excited level, ion and electron respectively.

Naturally, line radiation transitions are not considered here. Consistent with a two-level model, for radiative recombination we make the assumption that, except for the resonant continuum, all recombination processes lead to the excited modellevel (see also §4):

$$
k_{+1}^{\mathrm{r}}(T)=\sum_{i} k_{+i}^{\mathrm{r}}(T)
$$


This rate coefficient was obtained by numerical integration of

$$
\sum_{i} k_{+i}^{r}(T)=\frac{A}{T^{\frac{1}{2}}} \frac{g^{+}}{u_{+}(T)} \int \frac{\xi^{\mathrm{fb}}(\nu, T)[1-\exp (-h v / k T)]}{h v} \mathrm{~d} v
$$

At a temperature of $10000 \mathrm{~K},(10)$ gives

$$
2.9 \times 10^{-19} \mathrm{~m}^{3} / \mathrm{s} \text { (krypton) and } 3.7 \times 10^{-19} \mathrm{~m}^{3} / \mathrm{s} \text { (xenon). }
$$

The collisional-rate coefficients for the inverse processes are calculated from those of the direct processes, utilizing the method of detailed balancing. As usual, a linear cross-section law and a Maxwellian distribution of velocities have been assumed. The rate coefficients then are related to the cross-section constants $S^{k l}$ (see e.g. Demmig 1977), for which we have used the following values (those for xenon are in parentheses):

$$
\left.\begin{array}{ll}
S_{\mathrm{a}}^{01}=2.0 \times 10^{-5}\left(1.1 \times 10^{-4}\right) \mathrm{m}^{2} / \mathrm{J}, & S_{\mathrm{a}}^{1+}=0.5(0.5) \mathrm{m}^{2} / \mathrm{J} \\
S_{\mathrm{e}}^{01}=5.2 \times 10^{-3}\left(8.0 \times 10^{-3}\right) \mathrm{m}^{2} / \mathrm{J}, & S_{\mathrm{e}}^{1+}=4.0(4.0) \mathrm{m}^{2} / \mathrm{J} .
\end{array}\right\}
$$

As discussed in Fröbe, Müller \& Bötticher (1983), $S_{\mathrm{a}}^{01}$ and thus the relaxation time are strongly influenced by small degrees of impurities of the gas. However, at the end of the relaxation zone a variation of $S_{\mathrm{a}}^{01}$ only results in a time shift.

For deviations from a Maxwellian velocity distribution of the electrons and effects on the ionizational relaxation in shock waves see Meyer-Prüßner (1983). Close to the point of relaxation, however, and in the radiation-cooling region of interest here, deviations no longer persist because LTE conditions are approached.

The gasdynamical equations of one-dimensional unsteady flow read (Demmig 1977)

$$
\frac{\mathrm{D}_{0} \rho}{\mathrm{D} t}+\rho \frac{\partial u}{\partial x}=-R, \quad \rho \frac{\mathrm{D}_{0} u}{\mathrm{D} t}+\frac{\partial p}{\partial x}=0, \quad \rho \frac{\mathrm{D}_{0} h}{\mathrm{D} t}-\frac{\mathrm{D}_{0} p}{\mathrm{D} t}=-Q
$$

$u$ is the flow velocity, $p$ is the pressure and $\rho$ is the total mass density. The quantity $R$ in the equation of continuity describes the influence of the boundary layer in the shock tube (as a mass-loss term). $Q$ is the radiation energy loss defined in (7). The specific enthalpy $h$ contains the energy densities of excitation and ionization. Additionally, the equations of state for the ideal monatomic gas are used.

$T_{\mathrm{e}}$ is determined by the electron-energy equation, which balances the energy gain due to elastic electron-atom and electron-ion collisions and the energy loss originating from the inelastic processes. By detailed calculation and employing general rate equations (Murty 1971), the total radiation energy-loss term $Q$ of (7) can be shown to enter the electron-energy equation (Krauss-Varban 1981).

For the calculation of the elastic electron-atom collisional frequency we used the momentum transfer cross-section $Q_{\mathrm{ea}}^{\mathrm{m}}$ of Frost \& Phelps (1964), which in the case of krypton is multiplied by a factor of 2, according to the results of Müller (1981).

Because of the limitations of the common boundary-layer model used, which is based on a similarity assumption and transferred to a shock tube with quadratic cross-section, the quantity $R$ is multiplied by a correction factor $c_{R}$ close to unity, which has to be determined experimentally. Eventually, we hope to improve this description by detailed boundary-layer calculations. Unfortunately, in the case of unsteady flow, such calculations can only be done by investing considerable effort.

For the numerical calculation, the system of hyperbolic differential equations (12) is transformed into its characteristic normal form. It is solved with a first-order method of characteristics as described by Demmig (1978). The electron-energy 


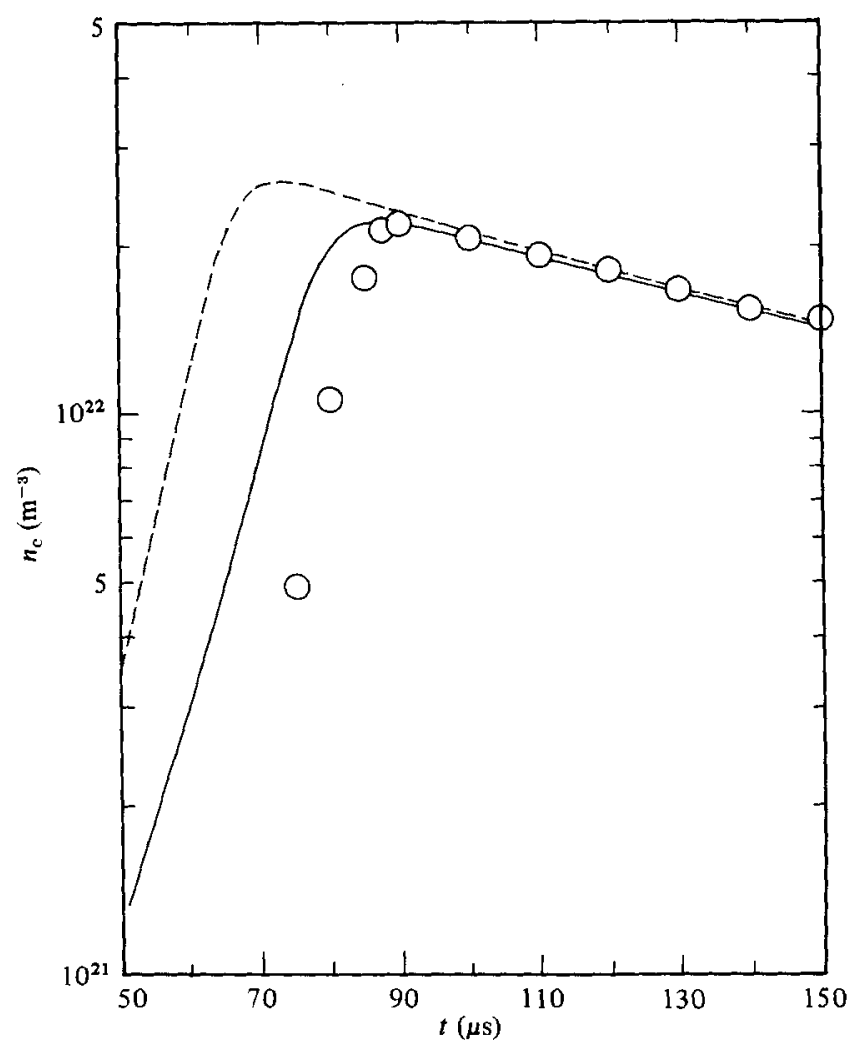

Figure 3. Computed electron-density profiles in krypton at two locations, separated by $1 \mathrm{~m}$. The solid curve corresponds to the location of measurement of the experimental data denoted by circles. Mach number 10.29 , test-gas density $0.0450 \mathrm{~kg} \mathrm{~m}^{-3}$, equilibrium temperature $9050 \mathrm{~K} ; a=1.6$, $c_{R}=1.8$, attenuation coefficient $1.3 \% \mathrm{~m}^{-1}$.

equation is solved by the secant method. The stiff rate equations require a special procedure: we used the method of Liniger \& Willoughby (1970) of first order and, alternatively, a method of second order reported by Steihaug \& Wolfbrandt (1979). The computation proceeds as indicated in Demmig (1978).

It appeared to be advantageous to improve the accuracy of the particle densities and electron temperature in one single iteration at every gasdynamical step. This is because of the strong coupling of the electron-energy equation and the rate equations. The method of Steihaug \& Wolfbrandt gave rise to $\frac{1}{5}-\frac{1}{10}$ shorter execution times than the first method; problems of stability did not appear. Giving an accuracy of about $2 \%$ in the particle densities, execution times amounted to between 1-10 s on a CD CYBER 76.

\section{Results and discussion}

The calculated electron-density time histories have been compared with experimental results obtained by two-wavelength interferometry (Schöpper 1976; and subsequent experimental work). When radiation energy loss is taken into account, a very good agreement of the electron densities results in the vicinity of the maximum and in the radiation cooling region (see figures 3-6; for the relaxation time see the comment on $S_{\mathrm{a}}^{01}$ in $\S 3$ ). On the average the theoretical maxima only show a deviation 

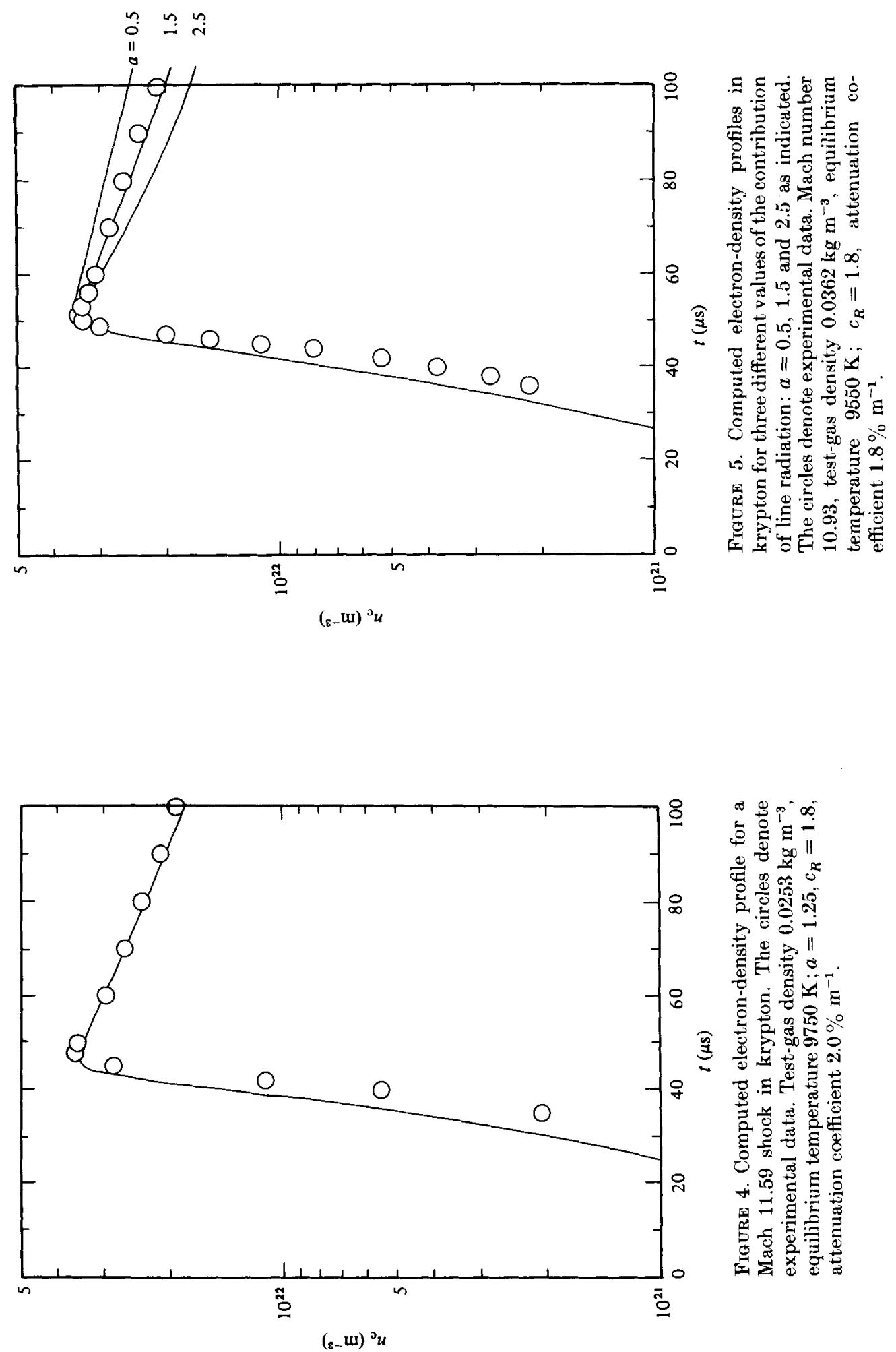


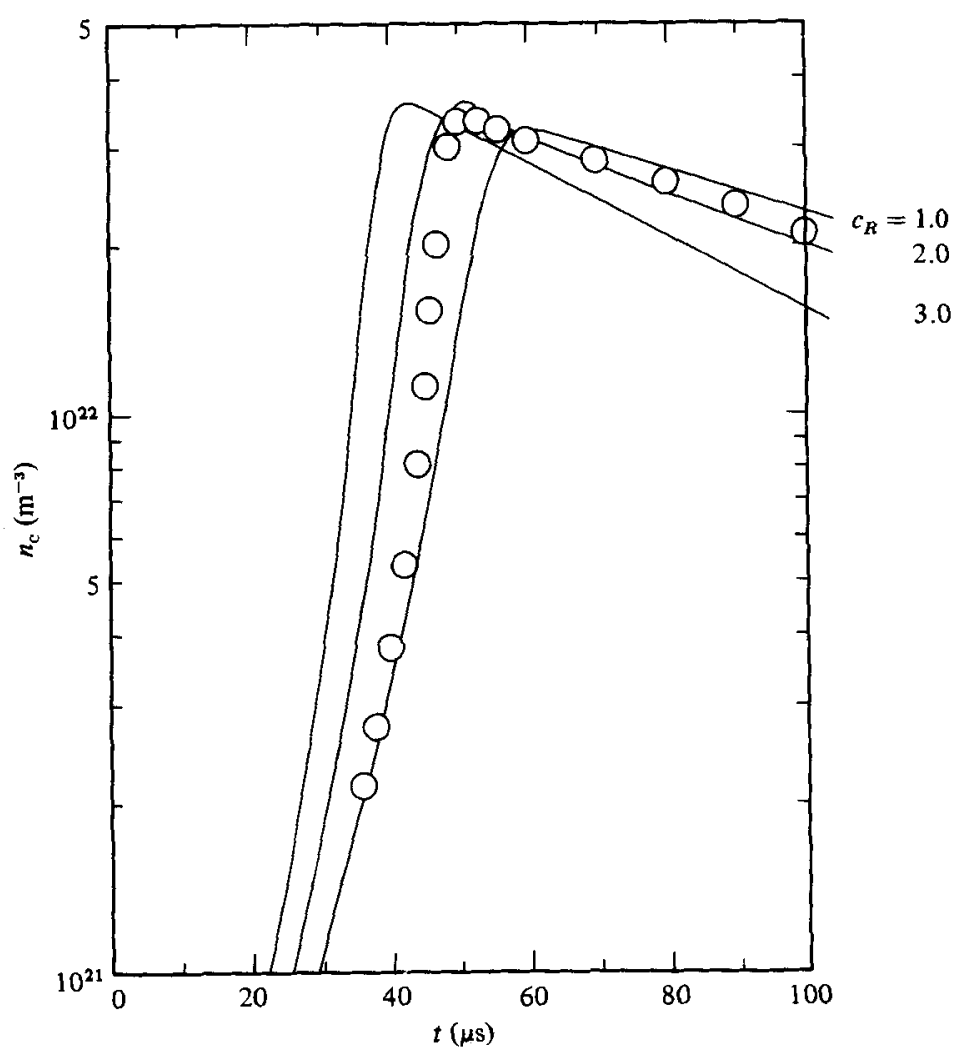

Figure 6. Computed electron-density profiles for three different values of the correction factor for the boundary layer: $c_{R}=1.0,2.0$ and 3.0 as indicated. Shock conditions and experimental data as in figure $5 ; a=1.4$.

of $\pm 2.5 \%$ from the experimental values. Larger discrepancies occur at later times at the measuring location ( $\gtrsim 100-150 \mu$ s after passage of the shock front), when the transition from a laminar to a turbulent boundary layer takes place and the boundarylayer model used becomes invalid (experiments by Stielow (1983, personal communication) using schlieren photographs support this assumption).

Dependent on the Mach number, the radiation shortens the relaxation time ( 0 for $M_{\mathrm{s}}=10,10 \%$ for $M_{\mathrm{s}}=12$ ) and causes a general decrease of the electron-density maximum of about $9 \%$ in krypton and $13 \%$ in xenon. Whereas the radiation-loss term in the electron-energy equation is important, the radiative-recombination rate only has a minor effect on $n_{1}$, and virtually no effect on $n_{\mathrm{e}}$.

Comparison of the electron densities at two different locations of measurement shows clearly the necessity of an unsteady description of real shock waves (see figure 3). Figure 5 shows a variation of the contribution $a$ of line radiation ; figure 6 exhibits the effect of the correction factor $c_{R}$ for the boundary layer. Both quantities influence the electron-density maximum as well as the radiative-cooling region.

A number of experiments in krypton have been evaluated to determine these free parameters. We approximated the calculated values of $\ln n_{\mathrm{e}}$ by a straight line in the radiative-cooling region and employed a least-square fit (see figure 2 for some resulting values of $a$ ). Using the relative temperature dependence $a(T)$ of figure 2, 
and also considering the absolute value of the electron-density maximum, we obtained

$$
c_{R}=1.8 \pm 0.2 \text { and } a= \begin{cases}1.60 & \text { at } T=9000 \mathrm{~K} \\ 1.15 & \text { at } T=10000 \mathrm{~K}\end{cases}
$$

equivalent to a contribution of line radiation to total radiation energy loss of $62 \%$ and $54 \%$, respectively.

Inspection of the electron gradient in the avalanche region supports these results. The value of $a$ is in fair agreement with the result of Glass et al. (1977), who obtained about $50 \%$ at temperatures around $11000 \mathrm{~K}$.

First comparisons of calculated electron densities with experimental values in xenon (Schwarz 1983) indicate $c_{R}$ being closer to unity, and a contribution of line radiation to total radiation energy loss similar to the result in krypton, contrary to the value obtained by Enomoto (1972). (For Mach number 10 Enomoto obtained $80 \%$, which amounts to $74 \%$, taking into account the different theoretical continuumradiation values his result was based on.)

We would like to thank W. Bötticher and the members of the shock-tube group of our institute for many helpful discussions and for providing their experimental results used in this work.

\section{REFERENCES}

Demmig, F, 1977 In Shock Tube and Shock Wave Research (Proc. 11th Intl Shock Tube Symp., Sealtle) (eds. Ahlborn, B., Hertzberg, A., Russel, D.) pp. 119-126.

Dеммія, F. 1978 Comp. Phys. Commun. 14, 7-12.

Enomoto, Y. 1972 J. Sci. Hiroshima Univ. А 36, 96-120.

Fröвe, U., Müllen, B.-H. \& Bötticher, W. 1983 J. Phys. B: Atomic \& Molec. Phys. 16, $4259-4271$.

Frost, L. S. \& Phelps, A. V. 1964 Phys. Rev. 136, A1538-A1545.

Geltman, S. 1973 J. Quantit. Spectrosc. Rad. Transfer 13, 601-613.

Glass, I. I. \& LiU, W.S. 1978 J. Fluid Mech. 84, 55-77.

Glass, I. I., LiU, W. S. \& TANG, F. C. 1977 Can. J. Phys. 55, 1269-1279.

Hofsäss, D. $1978 J$. Quantit. Spectrosc. Rad. Transfer 19, 339-352.

Horn, K. P., Wong, H. \& Bershader, D. 1967 J. Plasma Phys. 1, 157-170.

Krauss-Varban, D. 1981 Diplomarbeit, Universität Hannover.

Liniger, W. \& Willoughby, R. A. 1970 SIAM J. Numer. Anal. 7, 47-65.

MeYer-Prüssner, R. 1983 Dissertation, Universität Hannover.

MülleR, B.-H. 1981 Dissertation, Universität Hannover.

Murty, S. S. R. 1971 J. Quantit. Spectrosc. Rad. Transfer 11, 1681-1690.

Oettinger, P. E. \& Bershader, D. 1967 AIAA J. 5, 1625-1632.

Pinègre, M. \& Valentin, P. 1974 C. R. Acad. Sci. Paris B 279, 551-554.

Rehder, L., Bötticher, W., Carls, H. \& Graap, V. 1969 Phys. Fluids Suppl. 12, I50-I53.

SCHöpPER, W. 1976 Diplomarbeit, Universität Hannover.

Schwarz, H, 1983 Diplomarbeit, Universität Hannover.

Steihaug, T. \& Wolfbrandt, A. 1979 Math. Comp. 33, 521-534.

Vaguin, S. P., Yacobi, Yu. A., Yakovlev, V. V. \& Soloukhin, R. I. 1978 Rev. Phys. Appl. 13, $399-403$. 